\title{
High-Flow Nasal Cannula and Prone Positioning in Awake Patients with COVID-19 Related Respiratory Failure: An Observational Study.
}

Fekri Abroug ( $\square$ f.abroug@rns.tn )

Universite de Monastir Faculte de Medecine Dentaire de Monastir https://orcid.org/0000-0002-56265329

Zeineb Hammouda

CHU F.Bourguiba. Monastir

Manel Lahmar

CHU F.Bourguiba. Monastir

Wiem Nouira

CHU F.Bourguiba. Monastir

Syrine Maatouk

CHU F.Bourguiba. Monastir

Sourour Belhaj Youssef

CHU F.Bourguiba Monastir

Fahmi Dachraoui

CHU F.Bourguiba Monastir

Lamia Ouanes-Besbes

CHU F.Bourguiba Monastir

\section{Research}

Keywords: Covid-19, respiratory failure, HFNC, prone ventilation

Posted Date: November 1st, 2021

DOI: https://doi.org/10.21203/rs.3.rs-1023023/v1

License: (c) (i) This work is licensed under a Creative Commons Attribution 4.0 International License.

Read Full License 


\section{Abstract}

Background: We report an observational study on the use of High-flow nasal cannula (HFNC) and awake prone position in patients with Covid-19 related severe ARF.

Methods: chart analysis of consecutive patients with confirmed Covid-19 and severe ARF (PaO2/FiO2 ratio $<150$ ) who received HFNC. Patients were systematically encouraged to lie in the prone position if tolerated. We calculated initial ROX index (the ratio of SpO2/FIO2 to respiratory rate) while in supine position and at the end of the first HFNC session whether in prone or supine position, and their difference (delta ROX). The success/failure of HFNC (need for intubation) was recorded. Predictors of HFNC failure were identified using ROC curve and logistic regression.

Results: HFNC was administered to 213 out of 360 patients with COVID-19 related severe ARF (71\% male, median age:59 years (IQR:50-68), median Pa02/FIO2: 104(73-143). At the start of HFNC, median ROX index was 4(3.4-5). Among included patients, 178 (83.5\%) could tolerate prone position and had a median of 4.4(2-6) prone sessions during a median of 10(4-16) hours/day each, for a median of 4(2-7) days. Overall, HFNC failure occurred in 61 patients (28.1\%) with similar proportions in patients who had HFNC in prone position and in patients who did not tolerate prone position (29\% and $26 \%$, respectively; relative risk:1.14. 95\% Cl:0.62-2.1). In the prediction of HFNC outcome, AUC was highest for delta ROX (AUC=0.83); AUC for baseline ROX (0.71), PaO2/FiO2 (0.73), and SpO2 (0.67), were significantly lower. The delta ROX cut-off $\leq 1.8$ had the best Youden index indicating the best combination of sensitivity (0.89) and specificity (0.61) with a PLR (2.33) and a NLR (0.17) to predict HFNC failure. Logistic regression disclosed the following predictors of HFNC failure: delta ROX: $\mathrm{RR}=0.44,95 \% \mathrm{Cl}=0.32-0.62 ; \mathrm{p}-$ 0.0001); baseline ROX index: RR=0.58, 95\% Cl:0.39-0.85, $p=0.005$ ); SOFA score (RR=1.6 for each point; 95\%Cl: 1.1-2.2, $\mathrm{p}=0.007)$; and $\mathrm{PaO} / \mathrm{FiO} 2$ at admission: $\mathrm{RR}=0.96,95 \% \mathrm{Cl}=0.94-0.99)$. Prone position was not related to HFNC success.

CONCLUSION: Awake HFNC in prone position is feasible in most patients with severe hypoxemic COVID19. Indicators of ARF severity and the early response to HFNC, rather than prone position are independently associated with HFNC outcome.

\section{Introduction}

Acute respiratory failure (ARF) is an important clinical feature of COVID-19 pneumonitis leading to supplemental oxygen in $76 \%$ of hospitalized patients and ventilatory support in the majority of cases[13]. Predominant evidence prior to the onset of the Covid-19 pandemic favored the use of high flow nasal cannulas (HFNC) in de novo ARF instead of bilevel NIV[4] [5]. With the advent of Covid-19 pandemic, the so-called "aerosol-generating procedures" such as bilevel NIV and HFNC, were no longer recommended as first-line procedures because of the risk of dispersion of viral particles through droplets and aerosolized particles[6, 7]. However, a paradigm shift has gradually emerged recommending "avoidance of intubation" following both, observations on higher mortality associated with invasive ventilation (up to 
90\%)[8], and mitigation of the risk associated with "aerosol-generating procedures" by implemented precautions [9-11]. Recent reports show that the ventilatory support in Covid-19 related ARF usually consists in HFNC used in no-less than $55 \%$, bilevel NIV in $16 \%$, whereas invasive mechanical ventilation (IMV) is used in only $9 \%[3]$.

The new credit granted to non-invasive ventilation in the context of Covid-19 pandemic has favored the emergence of several studies aiming to assess the feasibility and the comparative performance of noninvasive oxygenation methods[1,12-16]. Grieco et al reported similar effects on the number of days free of respiratory support within 28 days in COVID-19 related moderate-to-severe hypoxemia, whether oxygenation relied on helmet NIV or HFNC [12]. A study falling within the framework of comparative effectiveness concluded to the superiority of oxygenation by CPAP over standard oxygenation, whereas HFNC was not better than the standard of care[1]. However, in these studies, patients were not ventilated in prone position which has been recently suggested to potentiate the effect of NIV in awake patients[1721]. In intubated as well as in spontaneously breathing patients, prone positioning improves indeed oxygenation and reduces the risk of lung injury. Increased oxygenation results from improved ventilation/perfusion matching through an improved ventilation in the well-perfused non-dependent areas of the lung [22-24]. The decreased risk of lung injury results from a decrease in chest wall compliance when the juxta-sternal part of the lung relies on the bed surface $[24,25]$. In a multinational meta-trial, Ehrmann et al have recently shown that in patients with hypoxemic respiratory failure due to Covid-19, awake prone positioning reduces the risk of treatment failure and the need for tracheal intubation[26].

Nonetheless, the availability of a clinical tool or a score that assists in making a rapid decision to forgo HFNC is of utmost importance to avoid any delay in the intubation decision and prognosis worsening [27, 28]. ROX index, corresponding to the ratio of oxygen saturation as measured by pulse oximetry/FiO2 to respiratory rate has been shown to identify patients at low risk for HFNC failure in patients with ARF and pneumonia $[29,30]$. However, there are little published data describing the use of ROX index to guide use of HFNC to treat COVID-19-associated respiratory failure[27, 31-33].

We report herein, the results of an observational study on the use of HFNC associated to prone positioning and the yield of ROX index in patients with Covid-19 related severe ARF.

\section{Methods}

This is an observational study with prospective data collection that lasted between September 02, 2020, and July 31, 2021. The study received the approval of the Institutional Review Board under the usual care label, which waived the need for individual consent.

\section{Study population}

charts of patients aged $\geq 18$ years consecutively admitted to the medical ICU for confirmed Covid- 19 and severe acute hypoxemic respiratory failure were analyzed. Covid-19 pneumonia was confirmed by positive pharyngeal/nasal swab rt PCR. Severe acute hypoxemic respiratory failure was diagnosed on the 
presence of dyspnea, tachypnea with respiratory rhythm $\geq 26$, tachycardia, an Sp02 $\leq 92 \%$ while breathing ambient air, and a PaO2/FiO2 ratio $<150$. Our ICU policy does not recommend bilevel NIV in hypoxemic respiratory failure with a preference to oxygenation with HFNC targeting a saturation range of $94-98 \%$. Patients already intubated at admission, or those requiring immediate intubation and invasive mechanical ventilation were not included in the study. Only patients who received HFNC were included in the study.

\section{Oxygenation Protocol:}

HFNC is started in supine position with AIRVO 2 (Fisher \& Paykel Healthcare) adapted with flowmeters to supply up to $60 \mathrm{I} / \mathrm{min}$ of humidified and heated oxygen through heated breathing tube (Air Spiral; Fisher \& Paykel Healthcare) and Optiflow interfaces (Fisher \& Paykel Healthcare). At HFNC start temperature is set at 33 to $37^{\circ} \mathrm{C}$ and the initial flow at $45 \mathrm{~L} / \mathrm{min}$ in order to fit patient's air demand. The flow might be increased up to $60 \mathrm{l} / \mathrm{min}$ according to patient's need and tolerance. The fraction of inspired oxygen (FiO2) concentration was set to maintain SpO2 levels $\geq 94 \%$.

All patients were instructed and supported to lie in the prone position daily for as long as was tolerated, ensuring they were predominantly on their chest. Patients who tolerated prone position were asked to remain in that position as long as possible on a 24-h basis. In patients who did not tolerate prone position, HFNC was administered in supine position.

HFNC treatment was continuous, with close monitoring of vital signs and respiratory pattern, and was maintained until either clinical improvement (defined as SpO2 >94\% without dyspnea or clinical signs of distress) allowing the passage of oxygen therapy through a reservoir at $15 \mathrm{Lpm}$ or lower according to Sp02 target[34], or clinical worsening mandating tracheal intubation Intubation and initiation of invasive mechanical ventilation was indicated when respiratory rate was above 30 breaths per min in association with respiratory muscle fatigue, respiratory acidosis ( $\mathrm{pH}$ below $7 \cdot 25)$, severe hypoxemia corresponding to SpO2 below $90 \%$ despite an $\mathrm{FiO} 2$ of $\geq 0 \cdot 8$ ), hemodynamic instability, or deteriorating consciousness level. HFNC failure was defined as the need for tracheal intubation and mechanical ventilation due to deterioration of respiratory function, or death of the patient.

Data Collection: The following information was collected in all patients on ICU admission: age gender, date of onset of symptoms and that of hospital admission, coexisting chronic morbidities.

Collected respiratory variables included peripheral oxygen saturation (SpO2), Respiratory Rate (RR), $\mathrm{PaO} 2 / \mathrm{FiO} 2$ ratio, allowing calculation of the ROX index as defined by the ratio of SpO2/FIO2 to respiratory rate, an index that has previously been validated as a predictor of HFNC success or failure[29]. These variables were recorded at admission (in supine position), and at the end of the first HFNC session (whether in prone or supine position in patients who did not tolerate awake prone position). The difference between ROX calculated at the end of the first HFNC session and that calculated at baseline (delta ROX) was calculated. 
The duration of the first HFNC session and the number of total HFNC sessions/patient were also recorded in each patient. The final outcome of HFNC (success or failure), the length of ICUstay, and patient's outcomes (hospital discharge, death) were recorded too.

\section{Statistical analysis:}

Data are presented as median with Interquartile range (IQR). Comparisons of clinical characteristics of patients were performed by Mann Whitney $U$ Test. Categorical variables are reported as numbers and percentages and analyzed using the Chi-squared test and Fisher's exact test. A $p$ value $<0.05$ was considered significant.

Receiver operating characteristic (ROC) curve analysis was performed to test the performance of variables such as SpO2 measured at admission on ambient air, baseline ROX index, delta ROX, and $\mathrm{PaO} 2 / \mathrm{FiO} 2$ in the prediction of HFNC outcome. Comparison of ROC curves relied on DeLong et al test[35]. Operative characteristics of various cut-off points were explored with the Youden index which combines sensitivity, specificity, and Likelihood ratios for rating diagnostic tests. Independent predictors of HFNC outcome were identified by Logistic regression which included variables that were significantly different between successful and failing HFNC groups. SPSS 21 for Windows was used for analysis.

\section{Results}

During the study-period, 360 patients with acute hypoxemic respiratory failure were admitted to our ICU (Figure 1: flow chart). Of these, 213 had oxygenation with HFNC and were included in the study. Despite the encouragement and support to lie in prone position, the procedure was not actually tolerated in 35 $(16 \%)$. The major limitations to prone positioning were related to back or shoulder pain $(47.1 \%)$, obesity (23.5\%), delirium (17.6\%), or refusal for general discomfort (11.8\%).

Baseline characteristics:

Table I depicts the main characteristics of patients included in the study. All patients (median age: 59(IQR:50-68), Male/Female ratio: 152/61, median BMI: 28(26-32), had confirmed molecular diagnosis of COVID-19 (positive rtPCR for viral RNA). They were admitted to the hospital at a median of 10(7-14) days following their first clinical manifestations of Covid-19 disease and transferred to the ICU at a median of 2(1-4) days after hospitalization. At admission, their median PaO2/FIO2 ratio was 104(73-143), and SpO2 on ambient air was $85(77-88)$. The median respiratory rate was $26(24-30)$ breaths/min, and the median ROX index when they started HFNC was 4 (3.4-5).

Table I: Baseline characteristics of patients who had prone HFNC according to their final status (success/failure) 


\section{All patients HFNC HFNC Failure \\ $\mathrm{n}=\mathbf{2 1 3} \mathrm{n}=61$ \\ $n=152$}

\section{Demographics/Morbidities}

Age, median (IQR), yr

$59(50-68)$

57(47-65)

64(55-71)

0.02

Sex (Male), $n(\%)$

152(71)

109(72)

44(72)

0.54

$B M I$, median (IQR), $\mathrm{Kg} / \mathrm{m} 2$

28(26-32)

28(26-31)

29(27-39)

0.09

Hypertension, $n(\%)$

$54(25)$

28(18)

26(43)

0.28

Diabetes mellitus, $n(\%)$

69(32)

46(30)

23(38)

0.45

\section{Severity}

Time-interval between first symptom and $\quad 10(7-14)$

hospitalization, days

Time-interval to transfer to ICU, days

2(1-4)

10(8-14)

10(7-14.5)

0.17

SAPS II, median (IQR)

27(26-32)

2(1-4)

$3(1.5-4.5)$

0.36

SOFA score, median (IQR)

$3(2-4)$

25(19-30)

32(27-38)

0.0001

SPO2 AA, median (IQR), \%

85(77-88)

$3(2-3)$

4(3-6)

0.0001

PaO2/FiO2, median (IQR)

104(73-143)

86(80-88)

80(70-87)

0.0001

HR (beats/min), median (IQR)

81(75-95)

114(82-160)

76(60-109)

0.0001

$R R$ (breaths/min), median (IQR)

26(24-30)

80(75-89)

85(80-106)

0.001

ROX Index at baseline, median (IQR)

4(3.4-5.2)

25(23-29)

30(25-35)

0.0001

$p H$

$7.45(7.42-$

7.48)

4.2(3.6-5.8)

3.5(3-4.2)

0.0001

$C R P, m g / l$

128(72-204)

7.45(

7.460

0.2

Leucocytes, median (IQR), /mm3

$9,470(7,500-$ $12,160)$

Lymphocytes, median (IQR), /mm3

$720(500-$

$1,075)$

$115(60-200)$

158(105-

216)

0.01

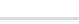

$9,500(7,500-$

$12,180)$

$9,400(7,050-\quad 0.96$

$12,110)$

Platelets/Lymphocytes, median (IQR)

Platelets/Lymphocytes, median (IQR)

$300(218-$

525)

800(500-

$1,100)$

$610(500-$

0.07

900)

Duration of stay, median (IQR), days

10(6-14)

292(225-

495)

309(192-

575)

0.9

9(6-13)

10(7-17)

0.08

Body position and HFNC Outcome: 
Overall, 178 patients (83.5\%) could tolerate prone position and had a median number of 4.4(2-6) prone positioning sessions which lasted a median of 10(4-16) hours/day each, for a median of 4(2-7) days oxygenation with HFNC. At the end of the first HFNC session which lasted a median of 6(4-9) hours, ROX index increased overall by a median of $1.62(0.45-3.9) ; p<0.0001$. In patients who tolerated prone position, the median increase in ROX index at the end of the first prone session was 1.67(0.83-3.5).

Overall, tracheal intubation (HFNC failure) occurred in 61 patients (28.1\%). Proportions of HFNC failure were similar in patients who had HFNC in prone position and in patients who did not tolerate prone position (29\% and 26\%, respectively; relative risk:1.14. 95\% Cl:0.62-2.1). Comparison between patients successfully managed with HFNC and those who failed shows that the latter group had significantly higher severity scores (SAPSII and SOFA), and lower levels of SpO2, PaO2/FiO2, and baseline ROX (Table I). ROX increase at the end of the first HFNC session (delta ROX) was significantly higher in the group of patients with successful HFNC treatment than in the patients with failing HFNC (medians, IQR): 2.7(-0.7$3.6)$ and $0.47(-4.3-2.9)$, respectively.

ROC curves were drawn to explore the performance of indicators of disease severity in predicting HFNC outcome. AUC was highest for delta ROX (AUC=0.83). In comparison, AUC for baseline level of ROX was:0.71 ( $p=0.04$ vs delta ROX), AUC for PaO2/FiO2 was0.73 ( $p=0.05$ vs delta ROX), and AUC for SpO2 on ambient air was 0.67 ( $p=0.003$ vs delta ROX ; Figure 2). The delta ROX cut-off $\leq 1.8$ had the best Youden index indicating the best combination of sensitivity (0.89) and specificity (0.61) with a Positive Likelihood Ratio of 2.33, and a Negative Likelihood Ratio of 0.17 to predict HFNC failure.

The Logistic regression identified the following variables as independent predictors of HFNC failure: delta ROX: OR=0.44 (95\%Cl=0.32-0.62; $p=0.0001)$; baseline ROX index: OR=0.58 (95\% Cl:0.39-0.85, $p=0.005)$; SOFA score: $\mathrm{OR}=1.6$ for each point; $95 \% \mathrm{Cl}$ : 1.1-2.2, $\mathrm{p}=0.007$ ); and $\mathrm{PaO} 2 / \mathrm{FiO} 2$ at admission: $\mathrm{OR}=0.96$, $95 \% \mathrm{Cl}=0.94-0.99)$. Prone positioning was not related to HFNC success. Overall, ICU mortality occurred in 52 patients $(24.4 \%)$.

\section{Dicussion}

In this observational study out of 213 patients with Covid-19 related severe hypoxemic respiratory failure and instructed to lie in the prone position while receiving HFNC, 35 patients did not tolerate prone position while the remaining 178 (83.5\%) had HFNC in awake prone position. Intubation (HFNC failure) was required in similar proportions in patients who tolerated prone position $(52 / 178 ; 29 \%)$ and in those who had HFNC in the supine position during their ICU stay $(9 / 25 ; 26 \%)$. Delta ROX corresponding to the difference between ROX index calculated at ICU admission and after the first HFNC session was the best predictor of the outcome of HFNC. A ROX cut-off $\leq 1.8$ had the best operative characteristics in predicting the failure of HFNC. HFNC outcome was independently associated with indicators of ARF severity (such as SOFA score, baseline $\mathrm{PaO2} / \mathrm{FiO2}$, or baseline ROX) and not with body position.

Since the start of the pandemics, Covid-related severe hypoxemia revealed a great challenge to intensivists, with evolving paradigm regarding the place of non-invasive ventilation in this setting. From 
the ban unanimously applied on NIV during the first wave, evidence has progressively emerged on the reduced risk incurred by health workers and environment contamination when airways are managed without intubation, authorizing therefore the use of different NIV tools [10, 15, 36-40]. In early ARF, bilevel NIV delivered through helmet interface, and HFNC are deemed more effective than bilevel NIV delivered via facemask [41]. CPAP has recently emerged as the preferred tool of oxygenation in hypoxemic Covid19 patients[1].

Combining prone position in awake patients under spontaneous ventilation whichever the tool, is an idea that progressively gained scientific ground $[12,17,19,20,42,43]$. Prone positioning is indeed a first-line intervention in patients invasively ventilated for severe ARDS leading to substantial reduction in mortality through both, oxygenation improvement and VILI reduction[25, 44, 45]. Its use in awake and spontaneously breathing patients with ARDS secondary to COVID-19 has recently been reported to improve oxygenation in few retrospective or small sample prospective cohorts[19, 20,43,46,47]. One of the mechanisms that could explain the beneficial effect of ventilation in prone position is the homogenization of the VA/Q mismatch as shown in a case report by Zarontonello et al [46].

The observational nature of our study does not allow reliable assessment of prone impact on hard outcomes such as HFNC success or ICU mortality. The lack of randomization precludes random assignment to prone position and does not exclude statistical imbalance in variables with outcome relevance between patients who did tolerate prone position and those who did not. However, studies of varying sizes can provide information on the magnitude of the benefits associated with the application of awake prone HFNC in hypoxemic Covid-19. Small recent studies on the use of prone position in spontaneously breathing patients with Covid-19 related hypoxemia reported lower success rates of HFNC: $34 \%$ in Zucman et al study, and $46.7 \%$ in Blez et al study, compared to $64 \%$ in our cohort [32, 48]. Conversely, in a retrospective cohort analysis of Covid-19 patients oxygenated with HFNC whether in supine or in prone position, Ferrando et al reported similar rates of intubation in both groups $(41 \%$ and $40 \%$, respectively) and no significant risk in 28-day mortality[49].

In a landmark multinational meta-trial, Ehrmann et al have recently provided the evidence for the superiority of awake prone positioning over supine, in Covid-19 related hypoxemic respiratory failure[26]. The authors pooled and analyzed in advance the individual observations of patients already included in prospective studies in progress spread across the world. These studies had in common the objective of evaluating the impact of delivery of awake prone HFNC in patients with Covid-19 acute hypoxemic respiratory failure. The meta-trial included 1,121 patients and reported a reduced HFNC failure rate (intubation or death) in patients assigned to awake prone positioning (40\%), compared to patients assigned to standard care (46\%; Relative risk 0.86 [95\% $\mathrm{Cl} 0.75-0.98])$. They recommended accordingly routine awake prone position in patients with COVID-19 who require HFNC. They even claimed that equipoise surrounding the prone positioning of patients treated with HFNC does no longer exist recommending therefore to stop ongoing RCTs on the issue as performing HFNC in supine position becomes no longer ethical. Although observational in its design, our study was conducted before the publication of the meta-trial by Ehrmann et al and raises no ethical issue. Moreover, our observation of no 
significant difference between HFNC in supine or in prone position should not be considered in contradiction with that of Ehrmann et al since $95 \% \mathrm{Cl}$ boundaries surrounding point estimates overlap. Several explanations might account for the lack of statistical significance in our study. First, the lack of randomization cannot exclude an imbalance in baseline risk in patients who tolerated and those who did not tolerate prone position. Second, we cannot exclude a type II error and an underpowered study. Third, the multinational character of Ehrmann's study which on one hand reinforces the result's extrapolation might in the other hand, introduce a center bias. Mexico (and France) provided the highest number of participants whereas Spain, Ireland, and Canada contributed only modestly to the study participants. Discrepancy in study protocols or in clinical practice for procedures that were not standardized by protocols (such as actual prone duration which varied from 1.6 hour in Spain to $8.6 \mathrm{~h}$ in Mexico) might have impacted to a certain extent the failure/success rates. Besides, given its sample size, the Mexican subgroup had the highest weight among included subgroups and substantially impacted the final metatrial result as it was the only positive trial per se. One might speculate that the effect of awake prone position is risk-dependent requiring therefore a risk-stratified analysis. In this connection, the lower failure rate reported in our study compared to that reported in the meta-trial by Ehrmann et al, might suggest a lower overall failure risk in our population compared to that of the meta-trial. However, the rate of HFNC failure reported in our study compares fairly with that usually reported outside the setting of Covid-19 (34.3\% in the meta-analysis by Rochwerg et al[4]), or in Covid-19 [3].

In addition to information on feasibility and outcome of awake prone in real life, our study provides information on predictors of HFNC outcome in the specific setting of Covid-19 related hypoxemia. Our study confirms the performance of the ROX index evaluated elsewhere outside the Covid-19 setting. We show that the variation of the ROX index between baseline and the end of the first HFNC session, rather than a unique baseline measurement, is the best indicator of HFNC outcome. An increase by less than a threshold of 1.8 appears to have the best operative characteristics to predict HFNC failure. Early following setting up oxygenation with HFNC, clinicians have a reliable tool on outcome prediction and decisionmaking.

The usual questions in matters of HFNC arise therefore: in what proportion failure occurs, and how to predict them early enough to avoid the loss of chance related to delayed intubation in these patients. Although non-randomized, our study could answer most these previously enumerated relevant questions. It allowed precision of the rate of ROX index proved effective in the prediction of HFNC failure. In patients ventilated either in prone or in supine position, the cut-off of ROX variation between baseline and the end of the first HFNC session had the best operative characteristics among other physiologic variables such as severity of hypoxemia or general severity scores (SOFA). Our study further clarified the impact of HFNC combined to prone position on patient's survival.

\section{Conclusions}

Awake HFNC in prone position is feasible in most patients with severe hypoxemic COVID-19. Indicators of ARF severity and the early response to HFNC, rather than prone position are independently associated 
with HFNC outcome.

\section{Declarations}

Ethics approval and consent to participate: The study received the approval of the Institutional Review Board under the usual care label, which waived the need for individual consent.

\section{Consent for publication :NA}

Availability of data and materials: The data sets used and/or analysed in the current study are available from the corresponding author on reasonable request.

Competing interests: The authors declare that they have no competing interests

\section{Funding: None}

Authors' Contribution: Conception and design: FA and LOB; data acquisition and interpretation: ZH, ML, WN, SM, SBY, FD; analysis and interpretation of data: FA, LOB, and FD; drafting the manuscript and revision for important intellectual content: FA, LOB, FD; final approval of the submitted manuscript: all authors. All authors read and approved the final manuscript and are accountable for all aspects of the work.

\section{References}

1. Perkins GobotR-Rc: An adaptive randomized controlled trial of non-invasive respiratory strategies in acute respiratory failure patients with COVID-19. MedRxiv 2021.

2. Docherty AB, Mulholland RH, Lone NI, Cheyne CP, De Angelis D, Diaz-Ordaz K, Donegan C, Drake TM, Dunning J, Funk S et al: Changes in in-hospital mortality in the first wave of COVID-19: a multicentre prospective observational cohort study using the WHO Clinical Characterisation Protocol UK. The Lancet Respiratory medicine 2021, 9(7):773-785.

3. Docherty AB, Harrison EM, Green CA, Hardwick HE, Pius R, Norman L, Holden KA, Read JM, Dondelinger F, Carson G et al: Features of 20133 UK patients in hospital with covid-19 using the ISARIC WHO Clinical Characterisation Protocol: prospective observational cohort study. Bmj 2020, 369:m1985.

4. Rochwerg B, Granton D, Wang DX, Helviz Y, Einav S, Frat JP, Mekontso-Dessap A, Schreiber A, Azoulay E, Mercat A et al: High flow nasal cannula compared with conventional oxygen therapy for acute hypoxemic respiratory failure: a systematic review and meta-analysis. Intensive care medicine 2019, 45(5):563-572.

5. Ricard JD, Roca O, Lemiale V, Corley A, Braunlich J, Jones P, Kang BJ, Lellouche F, Nava S, Rittayamai $\mathrm{N}$ et al: Use of nasal high flow oxygen during acute respiratory failure. Intensive care medicine 2020, 
46(12):2238-2247.

6. Alhazzani W, Moller MH, Arabi YM, Loeb M, Gong MN, Fan E, Oczkowski S, Levy MM, Derde L, Dzierba A et al: Surviving Sepsis Campaign: Guidelines on the Management of Critically Ill Adults with Coronavirus Disease 2019 (COVID-19). Critical care medicine 2020, 48(6):e440-e469.

7. Warrillow S, Austin D, Cheung W, Close E, Holley A, Horgan B, Jansen M, Joynt G, Lister P, Moodie S et al: ANZICS guiding principles for complex decision making during the COVID-19 pandemic. Crit Care Resusc 2020, 22(2):98-102.

8. Bahl A, Van Baalen MN, Ortiz L, Chen NW, Todd C, Milad M, Yang A, Tang J, Nygren M, Qu L: Early predictors of in-hospital mortality in patients with COVID-19 in a large American cohort. Internal and emergency medicine 2020, 15(8):1485-1499.

9. Tobin MJ: Basing Respiratory Management of COVID-19 on Physiological Principles. American journal of respiratory and critical care medicine 2020, 201(11):1319-1320.

10. Tobin MJ, Jubran A, Laghi F: P-SILI as justification for intubation in COVID-19: readers as arbiters. Annals of intensive care 2020, 10(1):156.

11. Tobin MJ, Laghi F, Jubran A: Caution about early intubation and mechanical ventilation in COVID-19. Annals of intensive care 2020, 10(1):78.

12. Grieco DL, Menga LS, Cesarano M, Rosa T, Spadaro S, Bitondo MM, Montomoli J, Falo G, Tonetti T, Cutuli SL et al: Effect of Helmet Noninvasive Ventilation vs High-Flow Nasal Oxygen on Days Free of Respiratory Support in Patients With COVID-19 and Moderate to Severe Hypoxemic Respiratory Failure: The HENIVOT Randomized Clinical Trial. Jama 2021, 325(17):1731-1743.

13. Duan J, Chen B, Liu X, Shu W, Zhao W, Li J, Li Y, Hong Y, Pan L, Wang K: Use of high-flow nasal cannula and noninvasive ventilation in patients with COVID-19: A multicenter observational study. The American journal of emergency medicine 2020.

14. Alqahtani JS: Global Current Practices of Ventilatory Support Management in COVID-19 Patients: An International Survey. Annals of intensive care 2020, 13:1635-1648.

15. Raoof S, Nava S, Carpati C, Hill NS: High-Flow, Noninvasive Ventilation and Awake (Nonintubation) Proning in Patients With Coronavirus Disease 2019 With Respiratory Failure. Chest 2020, 158(5):1992-2002.

16. Tverring J, Åkesson A, Nielsen N: Helmet continuous positive airway pressure versus high-flow nasal cannula in COVID-19: a pragmatic randomised clinical trial (COVID HELMET). Trials 2020, 21(1):994.

17. Tavernier E, McNicholas B, Pavlov I, Roca O, Perez Y, Laffey J, Mirza S, Cosgrave D, Vines D, Frat JP et al: Awake prone positioning of hypoxaemic patients with COVID-19: protocol for a randomised controlled open-label superiority meta-trial. 2020, 10(11):e041520.

18. Thompson AE, Ranard BL, Wei Y, Jelic S: Prone Positioning in Awake, Nonintubated Patients With COVID-19 Hypoxemic Respiratory Failure. JAMA internal medicine 2020, 180(11):1537-1539.

19. Elharrar X, Trigui Y, Dols AM, Touchon F, Martinez S, Prud'homme E, Papazian L: Use of Prone Positioning in Nonintubated Patients With COVID-19 and Hypoxemic Acute Respiratory Failure. Jama 2020, 323(22):2336-2338. 
20. Sartini C, Tresoldi M, Scarpellini P, Tettamanti A, Carco F, Landoni G, Zangrillo A: Respiratory Parameters in Patients With COVID-19 After Using Noninvasive Ventilation in the Prone Position Outside the Intensive Care Unit. Jama 2020, 323(22):2338-2340.

21. Jouffroy R, Darmon M, Isnard F, Geri G, Beurton A, Fartoukh M, Tudesq JJ, Nemlaghi S, Demoule A, Azoulay $\mathrm{E}$ et al: Impact of prone position in non-intubated spontaneously breathing patients admitted to the ICU for severe acute respiratory failure due to COVID-19. Journal of critical care 2021, 64:199-204.

22. Glenny RW, Lamm WJ, Albert RK, Robertson HT: Gravity is a minor determinant of pulmonary blood flow distribution. Journal of applied physiology 1991, 71(2):620-629.

23. Glenny RW, Polissar L, Robertson HT: Relative contribution of gravity to pulmonary perfusion heterogeneity. Journal of applied physiology 1991, 71(6):2449-2452.

24. Riad Z, Mezidi M, Subtil F, Louis B, Guerin C: Short-Term Effects of the Prone Positioning Maneuver on Lung and Chest Wall Mechanics in Patients with Acute Respiratory Distress Syndrome. American journal of respiratory and critical care medicine 2018, 197(10):1355-1358.

25. Gattinoni L, Taccone P, Carlesso E, Marini JJ: Prone position in acute respiratory distress syndrome. Rationale, indications, and limits. American journal of respiratory and critical care medicine 2013, 188(11):1286-1293.

26. Ehrmann S, Li J, Ibarra-Estrada M, Perez Y, Pavlov I, McNicholas B, Roca O, Mirza S, Vines D, GarciaSalcido $\mathrm{R}$ et al: Awake prone positioning for COVID-19 acute hypoxaemic respiratory failure: a randomised, controlled, multinational, open-label meta-trial. The Lancet Respiratory medicine 2021.

27. Procopio G, Cancelliere A, Trecarichi EM, Mazzitelli M, Arrighi E, Perri G, Serapide F, Pelaia C, Lio E, Busceti MT et al: Oxygen therapy via high flow nasal cannula in severe respiratory failure caused by Sars-Cov-2 infection: a real-life observational study. Ther Adv Respir Dis 2020, 14:1753466620963016.

28. Remy KE, Lin JC, Verhoef PA: High-flow nasal cannula may be no safer than non-invasive positive pressure ventilation for COVID-19 patients. Critical care 2020, 24(1):169.

29. Roca O, Caralt B, Messika J, Samper M, Sztrymf B, Hernandez G, Garcia-de-Acilu M, Frat JP, Masclans JR, Ricard JD: An Index Combining Respiratory Rate and Oxygenation to Predict Outcome of Nasal High-Flow Therapy. American journal of respiratory and critical care medicine 2019, 199(11):13681376.

30. Roca O, Messika J, Caralt B, Garcia-de-Acilu M, Sztrymf B, Ricard JD, Masclans JR: Predicting success of high-flow nasal cannula in pneumonia patients with hypoxemic respiratory failure: The utility of the ROX index. Journal of critical care 2016, 35:200-205.

31. Fink DL, Goldman NR, Cai J, El-Shakankery KH, Sismey GE, Gupta-Wright A, Tai CX: ROX Index to Guide Management of COVID-19 Pneumonia. Ann Am Thorac Soc 2021.

32. Zucman N, Mullaert J, Roux D, Roca O, Ricard JD, Contributors: Prediction of outcome of nasal high flow use during COVID-19-related acute hypoxemic respiratory failure. Intensive care medicine 2020, 46(10):1924-1926. 
33. Xia J, Zhang Y, Ni L, Chen L, Zhou C, Gao C, Wu X, Duan J, Xie J, Guo Q et al: High-Flow Nasal Oxygen in Coronavirus Disease 2019 Patients With Acute Hypoxemic Respiratory Failure: A Multicenter, Retrospective Cohort Study. Critical care medicine 2020, 48(11):e1079-e1086.

34. O'Driscoll BR, Howard LS, Earis J, Mak V, British Thoracic Society Emergency Oxygen Guideline G, Group BTSEOGD: BTS guideline for oxygen use in adults in healthcare and emergency settings. Thorax 2017, 72(Suppl 1):ii1-ii90.

35. DeLong ER, DeLong DM, Clarke-Pearson DL: Comparing the areas under two or more correlated receiver operating characteristic curves: a nonparametric approach. Biometrics 1988, 44(3):837-845.

36. Agarwal A, Basmaji J, Muttalib F, Granton D, Chaudhuri D, Chetan D, Hu M, Fernando SM, Honarmand $\mathrm{K}$, Bakaa $\mathrm{L}$ et al: High-flow nasal cannula for acute hypoxemic respiratory failure in patients with COVID-19: systematic reviews of effectiveness and its risks of aerosolization, dispersion, and infection transmission. Canadian journal of anaesthesia = Journal canadien d'anesthesie 2020, 67(9):1217-1248.

37. Li J, Ehrmann S: High-Flow Aerosol-Dispersing versus Aerosol-Generating Procedures. American journal of respiratory and critical care medicine 2020, 202(8):1069-1071.

38. Li J, Fink JB, Ehrmann S: High-flow nasal cannula for COVID-19 patients: risk of bio-aerosol dispersion. The European respiratory journal 2020, 56(4).

39. Fink JB, Ehrmann S, Li J, Dailey P, McKiernan P, Darquenne C, Martin AR, Rothen-Rutishauser B, Kuehl PJ, Haussermann S et al: Reducing Aerosol-Related Risk of Transmission in the Era of COVID-19: An Interim Guidance Endorsed by the International Society of Aerosols in Medicine. Journal of aerosol medicine and pulmonary drug delivery 2020, 33(6):300-304.

40. Tobin MJ: The criteria used to justify endotracheal intubation of patients with COVID-19 are worrisome. Canadian journal of anaesthesia = Journal canadien d'anesthesie 2021, 68(2):258-259.

41. Rochwerg B, Brochard L, Elliott MW, Hess D, Hill NS, Nava S, Navalesi PMOTSC, Antonelli M, Brozek J, Conti G et al: Official ERS/ATS clinical practice guidelines: noninvasive ventilation for acute respiratory failure. The European respiratory journal 2017, 50(2).

42. Li J, Pavlov I, Laffey JG, Roca O, Mirza S, Perez Y, McNicholas B, Cosgrave D, Vines D, Tavernier E et al: Meta-trial of awake prone positioning with nasal high flow therapy: Invitation to join a pandemic collaborative research effort. Journal of critical care 2020, 60:140-142.

43. Telias I, Katira BH, Brochard L: Is the Prone Position Helpful During Spontaneous Breathing in Patients With COVID-19? Jama 2020, 323(22):2265-2267.

44. Guerin C, Albert RK, Beitler J, Gattinoni L, Jaber S, Marini JJ, Munshi L, Papazian L, Pesenti A, Vieillard-Baron A et al: Prone position in ARDS patients: why, when, how and for whom. Intensive care medicine 2020, 46(12):2385-2396.

45. Guerin C, Reignier J, Richard JC, Beuret P, Gacouin A, Boulain T, Mercier E, Badet M, Mercat A, Baudin $O$ et al: Prone positioning in severe acute respiratory distress syndrome. The New England journal of medicine 2013, 368(23):2159-2168. 
46. Zarantonello F, Andreatta G, Sella N, Navalesi P: Prone Position and Lung Ventilation and Perfusion Matching in Acute Respiratory Failure due to COVID-19. American journal of respiratory and critical care medicine 2020, 202(2):278-279.

47. Xu Q, Wang T, Qin X, Jie Y, Zha L, Lu W: Early awake prone position combined with high-flow nasal oxygen therapy in severe COVID-19: a case series. Critical care 2020, 24(1):250.

48. Blez D, Soulier A, Bonnet F, Gayat E, Garnier M: Monitoring of high-flow nasal cannula for SARS-CoV2 severe pneumonia: less is more, better look at respiratory rate. Intensive care medicine 2020, 46(11):2094-2095.

49. Ferrando C, Mellado-Artigas R, Gea A, Arruti E, Aldecoa C, Adalia R, Ramasco F, Monedero P, Maseda $\mathrm{E}$, Tamayo $\mathrm{G}$ et al: Awake prone positioning does not reduce the risk of intubation in COVID-19 treated with high-flow nasal oxygen therapy: a multicenter, adjusted cohort study. Critical care 2020, 24(1):597.

\section{Figures}




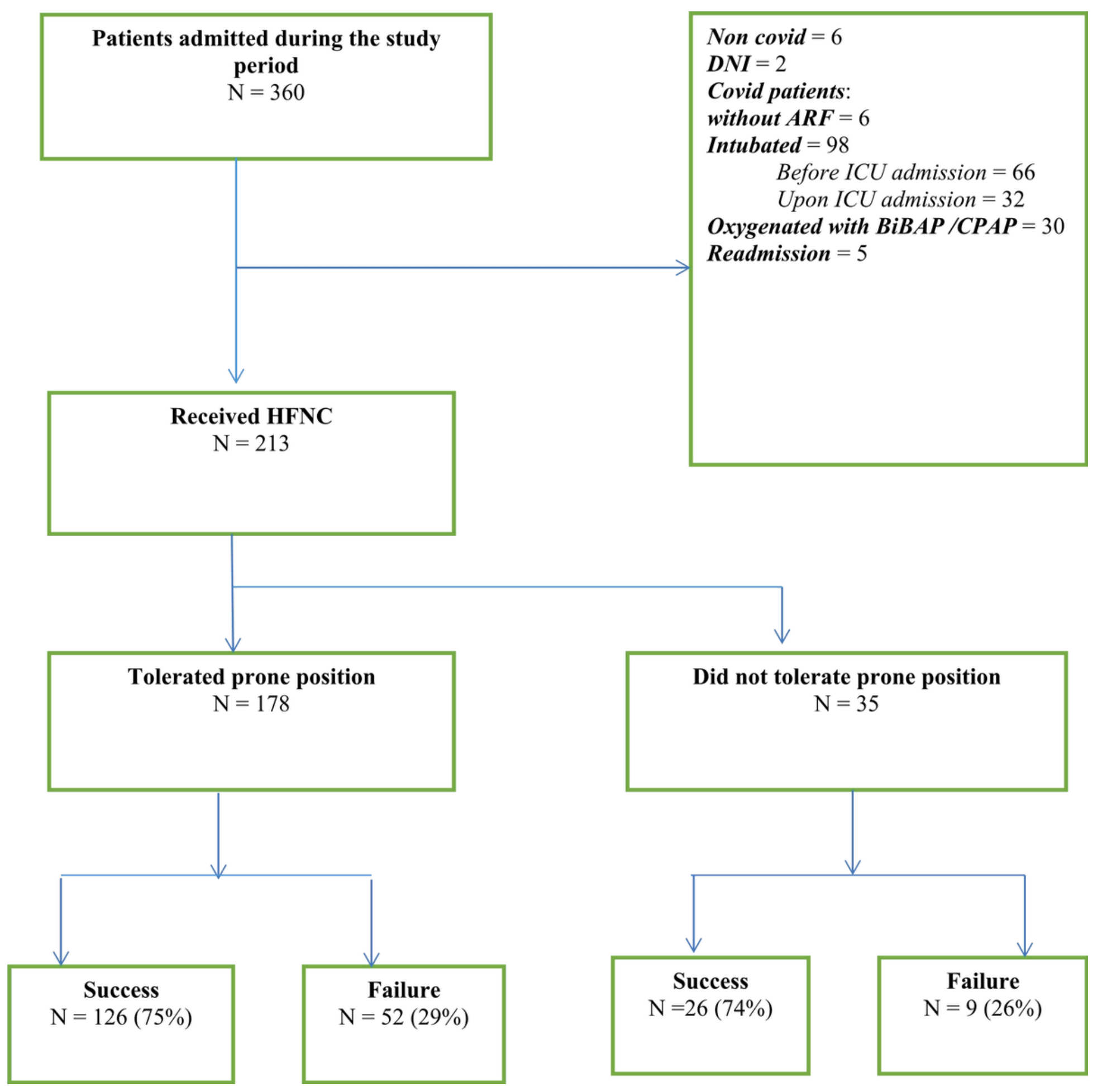

Figure 1

study flow chart 


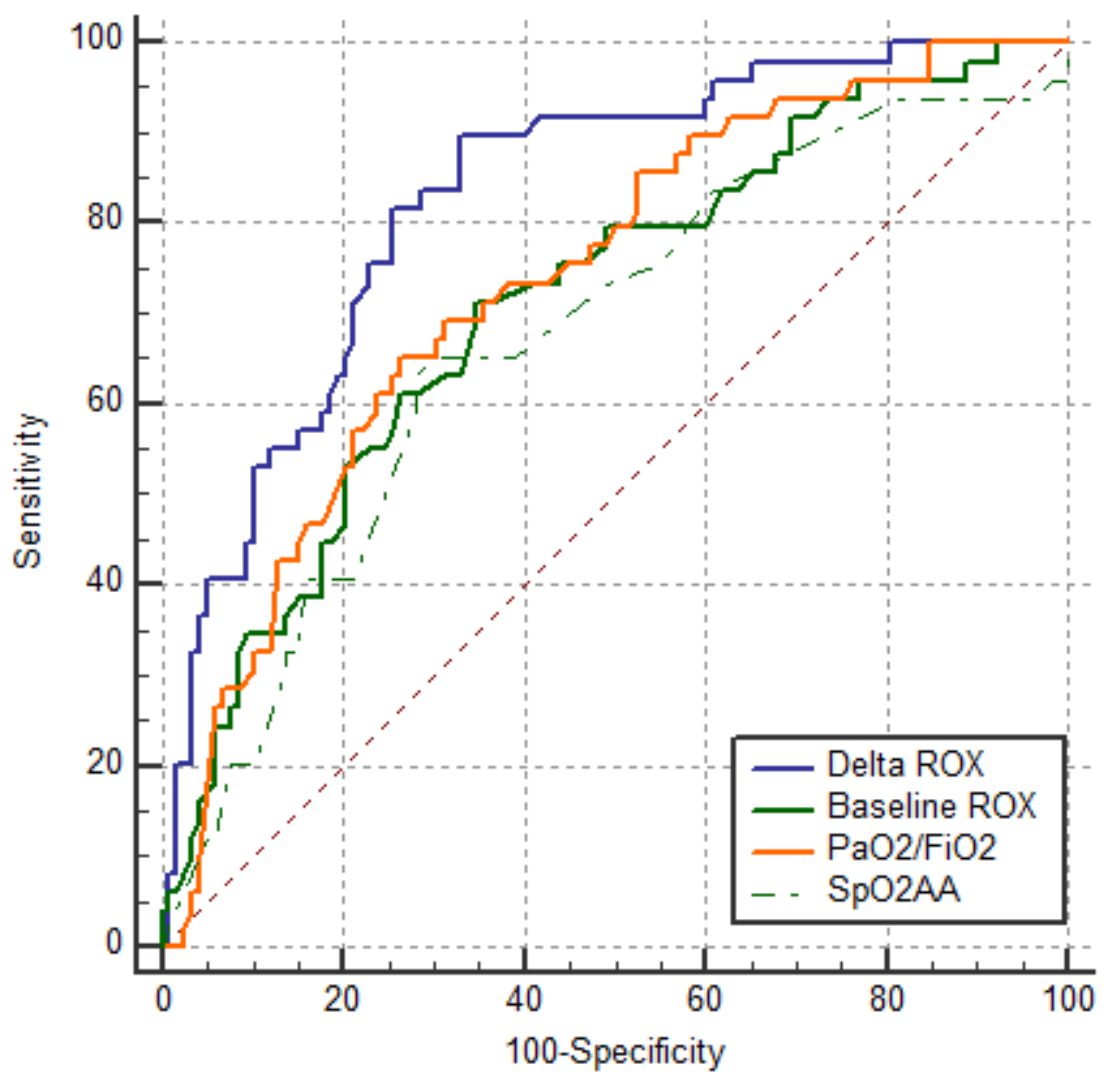

Figure 2

ROC curves of variables with potential for predicting HFNC success. AUC for delta ROX (AUC=0.83) was significantly higher than $A U C$ of baseline ROX ( $A U C=0.71 ; p=0.04$ vs delta ROX), baseline $\mathrm{PaO} / \mathrm{FiO} 2$ (AUC=0.73; $p=0.05$ vs delta ROX), and SpO2 on Ambient Air (AUC=0.67; $p=0.03$ vs delta ROX). 